\title{
Conceptual framework for research and clinical practice concerning cardiovascular health-related behaviors ${ }^{1}$
}

\author{
Maria Cecília Bueno Jayme Gallani ${ }^{2}$ \\ Marilia Estevam Cornélio ${ }^{3}$ \\ Rúbia de Freitas Agondi ${ }^{3}$ \\ Roberta Cunha Matheus Rodrigues ${ }^{4}$
}

Objective: To present a conceptual framework based on the PRECEDE model conceived to guide research and the clinical practice of nurses in the clinical follow-up of patients with cardiovascular diseases. Method: The conceptual bases as well as the study designs used in the framework are discussed. The contextualization of the proposed structure is presented in the clinical follow-up of hypertensive patients. Examples of the intervention planning steps according to the intervention mapping protocol are provided. Results: This conceptual framework coherently and rationally guided the diagnostic steps related to excessive salt intake among hypertensive individuals, as well as the development and assessment of specific interventions designed to change this eating behavior. Conclusion: The use of this conceptual framework enables a greater understanding of health-related behaviors implied in the development and progression of cardiovascular risk factors and is useful in proposing nursing interventions with a greater chance of success. This model is a feasible strategy to improve the cardiovascular health of patients cared for by the Brazilian Unified Health System.

Descriptors: Behavior; Health Planning; Health Education; Nursing.

\footnotetext{
${ }^{1}$ This research was supported by Fundação de Amparo à Pesquisa do Estado de São Paulo (FAPESP), process \# 2006/58789-5 e 2008/11596, Coordenação de Aperfeiçoamento de Pessoal de Nível Superior (CAPES), Fundação de Apoio ao Ensino, Pesquisa e Extensão - Unicamp, process \# 519.294.

2 PhD, Associate Professor, Faculdade de Enfermagem, Universidade Estadual de Campinas, Brazil. Associate Professor, Faculté des sciences infirmières, Université Laval, Québec, Canada.

${ }^{3}$ Doctoral Student, Faculdade de Ciências Médicas, Universidade Estadual de Campinas, Brazil.

${ }^{4} \mathrm{PhD}$, Associate Professor, Faculdade de Enfermagem, Universidade Estadual de Campinas, Brazil.
} 


\section{Estrutura conceitual para pesquisa e prática clínica na mudança de comportamentos em saúde cardiovascular}

Objetivo: apresentar a estrutura conceitual baseada no modelo PRECEDE, para guiar a pesquisa e a prática clínica do enfermeiro, na abordagem do paciente cardiopata ao longo de seu seguimento clínico. Método: as bases conceituais, assim como os desenhos metodológicos de pesquisa, implicados na estrutura conceitual, são abordados. A seguir, a contextualização da estrutura proposta é ilustrada no seguimento clínico de pacientes hipertensos, com incorporação das etapas de planejamento de intervenções, segundo o protocolo intervention mapping. Resultados: constata-se que a utilização dessa estrutura conceitual permitiu guiar, de modo coerente e racional, as etapas diagnósticas relacionadas ao consumo excessivo de sal entre os hipertensos, bem como norteou o desenvolvimento e avaliação de intervenções específicas para mudança desse comportamento alimentar. Conclusão: a utilização da estrutura conceitual proposta permite compreensão mais ampla dos comportamentos em saúde, implicados no desenvolvimento e progressão dos fatores de risco cardiovascular, assim como revela-se um caminho interessante para a proposição de intervenções de enfermagem, com maior chance de sucesso. O emprego dessa estrutura, de modo ampliado, pode constituir um meio factível para melhorar a saúde cardiovascular dos pacientes atendidos pelo Sistema Único de Saúde.

Descritores: Comportamento; Planejamento em Saúde; Educação em Saúde; Enfermagem.

\section{Modelo conceptual para la investigación y la práctica clínica en los cambios de comportamiento en salud cardiovascular}

Objetivo: presentar el marco conceptual basado en el modelo PRECEDE, concebido para orientar la investigación y la práctica clínica de las enfermeras para el seguimiento clínico de pacientes cardíacos. Método: La base conceptual, así como los diseños metodológicos de los estudios implicados en el marco conceptual son discutidos. Además, la contextualización del modelo se ilustra en el seguimiento clínico de los pacientes hipertensos. Ejemplos de las etapas de planificación de la intervención de acuerdo con el protocolo intervention mapping son proporcionados. Resultados: Se observó que la utilización del marco conceptual permitió guiar coherentemente y racionalmente los pasos de diagnóstico relacionados con el consumo excesivo de sal entre hipertensos, así como el desarrollo y la evaluación de las intervenciones específicas dirigidas a este comportamiento nutricional. Conclusión: El uso del marco conceptual propuesto permite una mayor comprensión de los comportamientos de salud implicados en el desarrollo y progresión de los factores de riesgo cardiovascular, así como se muestra una propuesta interesante para las intervenciones de enfermería con mayores posibilidades de éxito. El uso ampliado de este modelo puede ser un medio factible para mejorar la salud cardiovascular de los pacientes incluidos en el Sistema Nacional de Salud.

Descriptores: Conducta; Planificación en Salud; Educación en Salud; Enfermería.

\section{Introduction}

Cardiovascular diseases (CVDs) are the main cause of deaths and loss of quality of life due to related disabilities in the world(1). Even though a decline in mortality rates has been observed in recent decades, the impact of CVDs is still expressive. One out of three deaths that occurred in the United States between 1998 and 2008 was caused by CVDs. Actually, estimates indicate that more than 2,200 deaths per day (one death every 39 seconds) are caused by CVDs in the United States ${ }^{(1)}$. In Brazil, cardiovascular diseases are the primary cause of death. According to the Health Indicator and Basic Data in Brazil (IDB), cardiovascular diseases accounted for approximately 31\% (320,074 in absolute numbers) of deaths in $2009^{(2)}$. These data indicate the burden that CVDs represent for the Brazilian Unified Health System, reinforcing the need to develop effective strategic health interventions to reduce the manifestation of these diseases in Brazil.

One of the cardinal points in controlling CVDs is addressing cardiovascular risk factors, since the high 
incidence of risk factors is closely associated with the occurrence of this particular group of diseases. As initially shown in the study by Framinhgham(3) and confirmed in subsequent studies, risk factors are ubiquitous in the world, in different racial and ethnical groups, and is closely linked to the lifestyles of populations. It has been proven that the control of risk factors through specific programs results in reduced morbidity and mortality due to cardiovascular diseases and due to all other causes(4-6). A metanalysis revealed that regardless of strategies used to control risk factors, that is, whether the programs focus on educational strategies, physical conditioning or combined interventions, the results obtained are similar(6).

Activities included in cardiac rehabilitation programs, as well as in broader health promotion programs, are mainly educational interventions. Most of these actions are focused on changing health-related behaviors including also the clinical assessment and follow-up of those individuals with the manifestation of the diseases. These strategies can be successfully implemented by nurses due to their clinical competence, their holistic perspective regarding their patients, and their ability in the development of educational activities.

Given the magnitude of the problem related to CVDs in the Brazilian context, it is important that nurses use a conceptual structure that enables the planning and the assessment of interventions intended to reverse this situation through the control of risk factors and illness management. The use of rational intervention designs enables health educators to discover relationships, allocate resources and activities in certain fields and to evaluate the results of their actions(7). The more specific is each step of the planning process, the greater the chances are that the program will be effective in attaining the expected results.

This study's objective is to propose a conceptual structure that has guided our approach to research and clinical practice in the outpatient follow-up of individuals with cardiovascular diseases. Aiming to explore the applicability of this conceptual model, we contextualize its use in the specific follow-up of patients with hypertension. The use of a planning protocol for health education interventions - intervention mapping - is highlighted as well as the methodological research designs used in the conceptual model.

\section{A conceptual structure}

The structure of the conceptual model was based on the PRECEDE ${ }^{(8-10)}$ model with some adaptations ${ }^{(7)}$. The PRECEDE model recommends analyzing the cause of health problems at multiple levels, considering the multiple determinants of health and environment-related behavior. It is a revised and enlarged(9-10) framework to epidemiological population-based planning( ${ }^{(8)}$ widely used in the planning of interventions in several health educational programs ${ }^{(7)}$.

The PRECEDE model assumes the existence of personal and external factors that determine healthrelated behaviors. Environmental factors are seen as potential determinants of behavior. Behaviors are presented as determinant of health problems, which in turn influence the studied population's quality of life $\mathrm{e}^{(8-9)}$ (Figure 1).

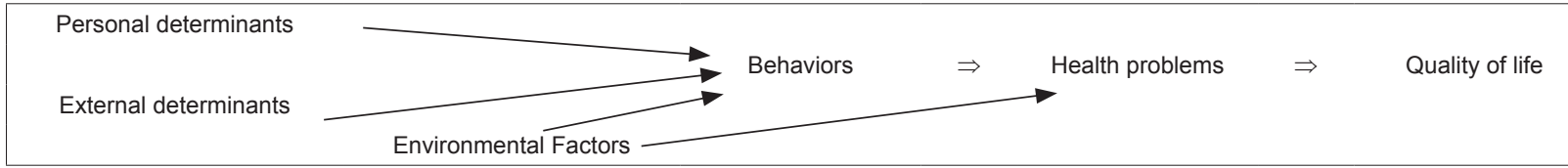

Figure 1 - Relationships among behavior, health problems, quality of life, and determinants. Adapted from the PRECEDE model ${ }^{(8-10)}$

The PRECEDE model allows to make a portray of the health problem in which one desires to intervene, which is the first of the six steps of the program-planning framework (intervention mapping)(7): evaluation of needs (diagnostic step). Based on the situational diagnosis, the following steps take place: proposing the matrix of change objectives, selection of the most appropriate theory based methods and practical strategies, program design, adoption and implementation plans, and finally, the results assessment.

In the model that we propose to the approach of hypertensive patients (Figure 2), we establish the hypothesis that causal relationships exist between the individual and social environmental factors that predispose, facilitate and reinforce hypertension-related behaviors (exercise, nutrition, medication therapy 
adherence). In turn, such behaviors have a causal relationship with the development and progression of cardiovascular risk factors and, consequently, with how severe hypertension is clinically expressed. The phenotypic expression of hypertension includes pressure levels, target organ damage, and progression to heart failure. It is assumed that genetic factors permeate the relationships among these variables and are believed to have a modulator role in the way and how intense responses to the individual-environment interaction are in terms of the disease's phenotypic expression.

The relationship of quality of life with the remaining variables distinguishes the structure we propose from study of the context of hypertensive patients, and the PRECEDE model. When causal relationships are investigated in the PRECEDE model, quality of life is considered a consequence of health-related problems. While in our model we assume that quality of life is a consequence of health-related problems, we also believe it intermediates the relationship among the remaining variables. In addition to the initial diagnosis of quality of life, we need to investigate the factors that are related to good or poor quality of life, as well as whether the different dimensions of quality of life have previously influenced health-related behavior and, consequently, influenced the final outcomes of health quality, the severity of disease and, finally, health-related quality of life (HRQOL).

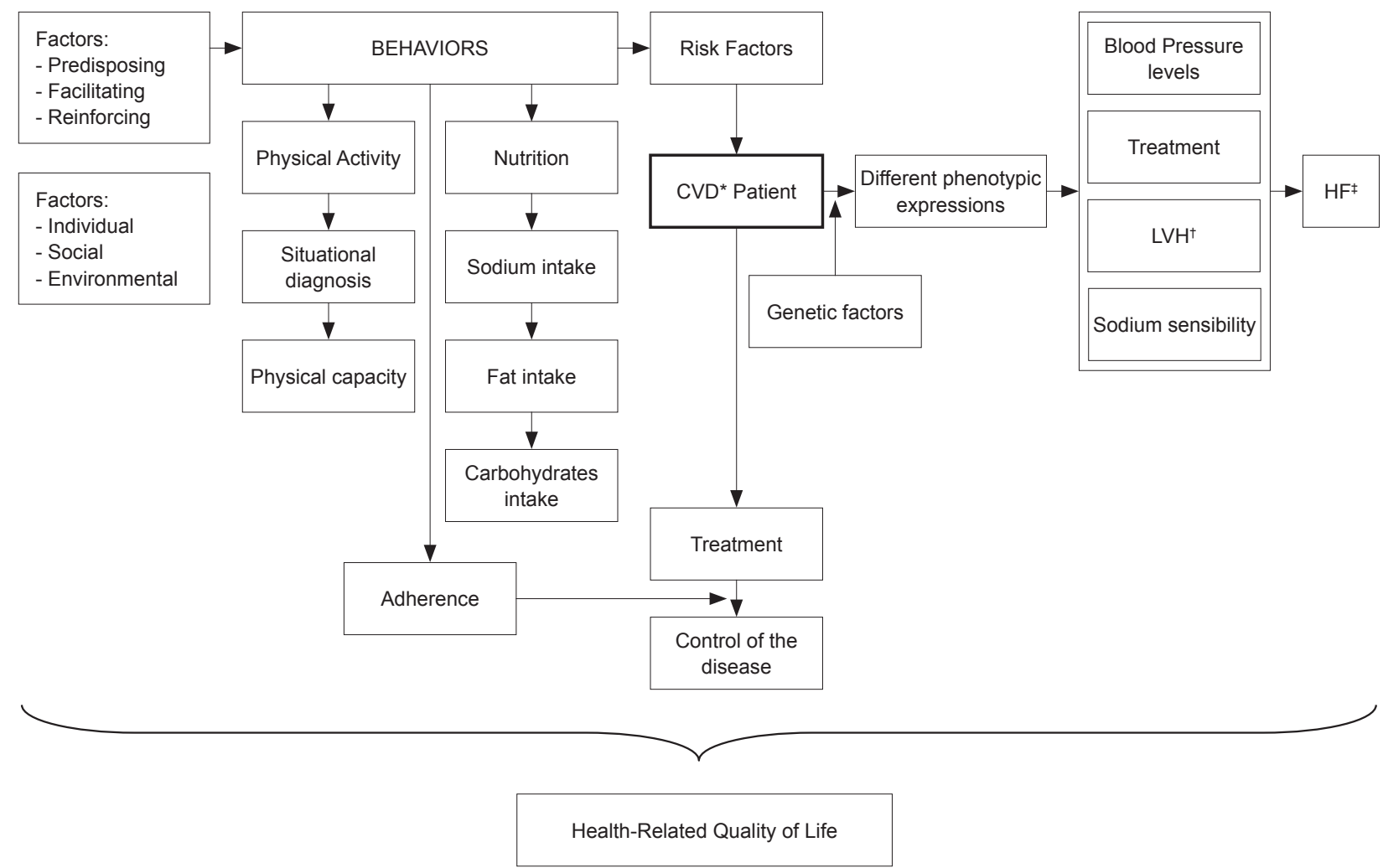

$* \mathrm{CVD}=$ Cardiovascular disease; $\mathrm{HLVH}=$ left ventricular hypertrophy; $\neq \mathrm{HF}=$ heart failure

Figure 2 - Representative scheme of the Conceptual Model

The advantage of developing this structure for diagnostic evaluation resides in the establishment of a logical model that indicates potential causal relationships among variables, enabling the employment of specific strategies for a situational diagnosis. Based on a directed diagnosis we can determine the objectives to be achieved. With diagnosis and objectives established, the selection of an intervention method becomes more objective, as does the criteria according to which the effectiveness of interventions will be assessed.

Another positive aspect of this model is the possibility of expanding exploratory investigations. In addition to the assumed causal relationships, new relationships can be investigated among the variables, broadening understanding of the context and, consequently, possibilities of addressing hypertensive patients. 
From the researcher's point of view, this framework enables planning a line of research where collaboration, financing, as well as the inclusion of students at different levels of research-scientific initiation, Master's and doctoral research-are scheduled.

\section{The conceptual structure and methodological designs}

The three major methodological research designsmethodological, exploratory (cross-sectional and longitudinal) - are needed in the proposed conceptual structure. The diagnostic step, or identification of needs, is a mandatory step in planning interventions ${ }^{(7)}$ and requires two methodological designs: methodological and exploratory.

An effective planning program should be directed to a real problem or need, which is defined as a discrepancy between what is and what would be expected for a certain group in a given situation ${ }^{(7)}$. In the case of health problems, the assessment of needs seeks to understand the real situation and compare it to what would be more desirable in terms of quality of life and health condition. The assessment also identifies the factors that influence health, such as behavior and environment. Planning is needed before initiating the identification of problems, including the establishment of the most appropriate tool to establish a diagnosis. The ideal tool is not, however, always available for the population of interest. Therefore, methodological studies are required to develop or adapt current instruments in other cultures.

Measuring psychosocial variables such as quality of life and individual factors that influence the motivation to adopt health-related behaviors (e.g. beliefs, attitudes and values) is not an easy task. These variables, called constructs due to their hypothetical and abstract nature, can, however, be measured as long as the instrument used is developed with the methodological rigor required. The quality of the instruments' measures as well as the correct interpretation of results should be clearly presented. Measuring health-related behavior itself is also complex and can be achieved through both objective and subjective measures. Both measures present advantages and limitations. The selection or the development of an instrument should be guided not only by the quality of its measures but also by how well it represents a group of interest and its specificity in relation to what is being studied.

Once the tools required for the identification of problems are obtained, the diagnosis process begins.
There is no specific order to how this step is initiated and the diagnosis may occur on multiple fronts, exploring the relationships among the involved variables so that the objectives to be obtained via the interventions can be more precisely established(7). Exploratory or predictive studies, e.g. cross-sectional or longitudinal studies, are preferred in this step in order to investigate relationships among variables and to determine the predictive abilities of some variables. Many studies were performed by our research group on the proposed conceptual structure to address hypertensive patients. These studies first focused on the development and refinement of measurement instruments and, later, exploratory studies focused on understanding the relationships among the model components, such as quality of life, symptoms, and clinical and genetic profiles. Predictive studies were used to identify determinants of healthrelated behaviors ${ }^{(11-14)}$

Once the diagnostic step is concluded, experimental studies are implemented. These studies are based on problems identified in the diagnostic step and propose, implement and assess the results of specific interventions. The evaluation of results that are obtained in experimental studies developed with sound methodological designs is essential to providing feedback on the relationships hypothesized among variables and to understand an intervention's potential mechanisms of action, which enable restructuring the intervention or recommending its use on a larger scale.

\section{Application of intervention mapping to reduce salt intake among hypertensive patients}

This section presents one of the research lines based on the conceptual model, on addressing the problem of excessive salt intake among hypertensive patients, a problem that emerged in our clinical practice concerning outpatient follow-up. We observed during nursing consultations while clinically assessing patients that they presented salt intake greater than what is recommended ( $4 \mathrm{~g}$ a day) by Brazilian and international guidelines for hypertensive individuals. Excessive salt intake directly and indirectly influences the phenotypical expression of hypertension, worsening its consequences and influencing responses to medication therapy. Hence, it was crucial to establish a precise diagnosis of the problem and develop an intervention intended to reduce sodium intake in this group of patients. The intervention mapping protocol was used to guide the intervention's development and assessment. 
Step I: identification of needs (diagnosis)

In addition to the fact that excessive salt intake is a key factor in the manifestation of cardiovascular diseases, especially hypertension, it has been associated with non-cardiovascular diseases, such as gastric cancer, osteoporosis, and obesity, among others(15-17). Excessive salt intake is currently considered the seventh most important cause of premature death in developed countries and the second in developing countries(18). Such evidence ratifies the existence of a real nutritional problem in the studied population and requires specific actions to encourage healthy salt consumption. However, measuring the magnitude of the problem is necessary, precisely quantifying sodium intake as well as identifying the major sources of sodium. Hence, the initial step consisted of developing self-report instruments that enabled such measurements. The first of a series of studies addressing this eating behavior was performed. It presented the development and validation of self-report measures of salt intake. These measures were validated through correlation tests with 24-hr urinary sodium excretion, which is considered the gold standard for salt intake(19). A disadvantage of this objective measure is that it reflects one-time food consumption and does not identify the food sources, which is only possible through self-report tools. In addition to validating self-report measures, this study also revealed very high salt intake-about $12 \mathrm{~g}$ salt/a day - among the participants, three times greater than the value recommended for hypertensive individuals ${ }^{(19)}$.

In this diagnostic step, a second study was performed ${ }^{(20)}$. This study aimed to identify the individual determinants of salt intake through the investigation of three eating behaviors: adding salt while preparing food, consuming high salt content foods, and using salt shakers at the dinning table. The Theory of Planned Behavior (TPB $)^{(21)}$, extensively used in studies designed to predict health behaviors that include eating behavior, was employed as the theoretical framework. According to the TPB, motivation to act is the main determinant of action (behavior) and motivation, in turn, is determined by attitude (behavior favorability), social standards (perceived social pressure to adopt behavior) and perceived behavioral control (the extent to which the individual perceives being in control of his/her own behavior). Perceived control can be an additional predictor of behavior. Other variables were also added to the model (self-efficacy and habit) in order to improve its prediction power considering their relevance in the studied context of eating behavior. We found that motivation explained $22 \%$ of the variability in adding salt while preparing food and motivation for this behavior was explained by the variables self-efficacy and habit(20).

Evidence found in previously mentioned studies ${ }^{(19-20)}$ grounded step 1 of the intervention mapping: salt intake is high among hypertensive individuals and the main source of consumption is adding salt while preparing food. A reduction in adding salt is explained in $22 \%$ of the cases by motivation (intention to act), which in turn is explained by the constructs of self-efficacy and habit.

Step II: establishment of the matrices of goals

The performance objectives were established taking into account the determinants of motivation to act (selfefficacy and habit) and the gap between intention and behavior (even though statistically significant, motivation explained only $22 \%$ of behavior variability). Hence, two main objectives were established and grounded two experimental studies. One of the studies, characterized as Motivational Intervention, aimed to reinforce the motivation to limit salt intake while preparing food and focused on perceived self-efficacy and on reducing the automatism (habit) of this nutritional behavior(22-23). The other study, characterized as Planning Intervention, focused on the activation of intention, that is, on increasing the realization of positive intention ${ }^{(24)}$. Both studies composed the Programa SALdável [Salthealth Program], which is intended to reduce salt intake among hypertensive patients.

Step III: selection of the theory-based method for practical strategies

After an extensive literature review and discussion among the members of the research group and experts concerning Motivational Intervention, we chose the consciousness-raising and counterconditioning methods (that originated in the transtheoretical model) to reduce habit and chose guided practice with feedback and identification of barriers and coping strategies (that originated from the cognitive-social model) to increase self-efficacy perception. Based on these methods, the techniques most appropriate to the context were selected: group activities that included brainstorming, use of practical activities with the use of measuring spoons, reading labels, use of figures and recipe books with natural seasonings to replace salt, the development of skills to limit salt intake, and role playing(23).

The implementation intention ${ }^{(25)}$ strategy was chosen for the study characterized as Planning 
Intervention. This technique consists of individual activities designed to develop action planning and to shield striving for goals from anticipated obstacles. The individual develops at least three plans of how, where, and with whom to implement the planned behavior. Then the individual is asked to anticipate three likely obstacles that may hinder the achievement of such a goal and establish a plan to overcome these obstacles(24-25).

Steps IV and V: program design and the intervention adoption and implementation plan

Details of the program design and its implementation can be found in the respective studies ${ }^{(22-24)}$.

\section{Step VI: Assessment}

The effectiveness of interventions was assessed by comparing responses among the control and experimental groups. The primary endpoint was the self-reported frequency with which salt addition was reduced while preparing food, a behavior specifically addressed in the intervention. The secondary endpoints included the objective measure of salt intake taken in 24-hr urinary excretion, quantity of salt intake per capita/mo. for the household, the use of ready-to-use seasonings per capita/mo. for the household, and the total of added salt (which corresponded to the sum of the two previous measures), in addition to measures of psychosocial variables: intention, self-efficacy and habit. At the end of the follow-up period, the experimental groups were debriefed in order to identify the intervention's positive points and limitations from the participants' perspectives. Both interventions were effective in reducing the addition of salt when preparing food compared to the control group. The result was positive for the primary endpoint and for the remaining measures of salt intake used as a secondary endpoint, except for the sodium urinary excretion, which presented borderline statistical significance in the group of Motivational Intervention. Assessment of the primary endpoint indicated the interventions were effective. In regard to the psychosocial variables, as expected, the Motivational Intervention improved the perception of self-efficacy, reduced the perception of behavior automatism (habit), and significantly increased motivation to limit salt intake. The Planning Intervention had no effect on self-efficacy and intention, which was also an expected result, since it did not aim to change the perception of self-efficacy or increase motivation but transform positive motivation into effective behavior. The Planning Intervention did reduce the perception of automatism, indicating that planning is linked to reduced automatic use of salt when preparing food. Data show that the interventions share the same mechanism of action, reducing the habit of salt consumption.

There is still an extensive range of analyses that can be performed based on the obtained data: a comparison of cost-efficacy relationship between the two interventions, analysis of potential mediator or moderator variables in the responses to the intervention, analysis of the characteristics of those who responded positively to the intervention and those who did not change their behavior, as well as content analysis of the debriefing sessions. Such information is valuable to evaluate interventions as a whole and implement the necessary adjustments so that interventions can be reproduced on a large scale. Further studies with diversified population strata should provide other indicators that can enable understanding of the intervention effect and contribute to its improvement. Subsequent studies can evaluate the effect of interventions in the medium and long terms concerning salt intake and evaluate the potential maintenance of the effect initially attained. Such studies can also analyze the effect of different levels of salt reduction on health outcomes, such as the clinical manifestation of hypertension and HRQOL.

\section{Final Considerations}

The conceptual structure proposed in this paper is intended to serve as a map to guide nurses in clinical practice when monitoring patients with a chronic disease such as cardiovascular diseases. The model assumes there is a complex interrelationship among the variables to be considered in the clinical followup of patients with cardiovascular diseases, as well as the incorporation of a systematic protocol to plan the educational interventions of nurses designed to modify health behaviors. Such interventions are closely related to the clinical manifestation of disease and HRQOL. Its applicability is broad and can be adapted to a large variety of health-related behaviors. A systematically planned implementation of interventions enables better allocation of resources and the optimization of results, improving care provided to patients with cardiovascular diseases and improving the chances of attaining positive outcomes. Thus, such a structure is useful in the proposition of large scale nursing interventions and is also a feasible strategy to improve the cardiovascular health of patients cared for by the Unified Health System. 


\section{References}

1. Roger VL, Go AS, Lloyd-Jones DM, Benjamin EJ, Berry JD, Borden WB et al. Executive summary: heart disease and stroke statistics-2012 update: a report from the American Heart Association. Circulation. 2012;125(1):188-97.

2. Datasus (BR). Indicadores e Dados Básicos - IDB. Brasil 20010 [base de dados na internet].Brasília; 2012 [acesso 5 agoo 2012]. Disponível em: http://tabnet. datasus.gov.br/cgi/tabcgi.exe?idb2010/c08.def

3. National Heart, Lung, and Blood Institute. Profile of the Framingham Heart Study. 2007 [acesso 5 ago 2011]. Disponível em: http://www.framingham.com/ heart/profile.htm

4. Stanek EJ, Sarawatec C, Willey VJ, Charland SL, Cziraky MJ. Risk of cardiovascular events in patients at optimal values for combined lipid parameters. Curr Med Res Opin. 2007;23(3):553-63.

5. Jones $\mathrm{PH}$. Clinical significance of recent lipid trials on reducing risk in patients with type 2 diabetes mellitus. Am J Cardiol. 2007;99(44A):133B-40B.

6. Clark AM, Hartling L, Vandermeer B, McAlister FA. Meta-analysis: secondary prevention programs for patients with coronary artery disease. Ann Intern Med. 2005; 143:659-72.

7. Bartholomew LK, Parcel GS, Kok G, Gottlieb NH, Fernandez ME. Planning Health Promotion Programs: An Intervention Mapping Approach. 3rd ed. San Francisco: Wiley; 2011.

8. Green LW, Kreuter MW. Health promotion planning: an educational and environmental approach. 2 ed. Mountain View: Mayfield; 1994.

9. Green LW, Kreuter MW. Health promotion planning: an educational and environmental approach. 3 ed. Mountain View: Mayfield, 1999.

10. Green LW, Kreuter MW. Health promotion planning: an educational and environmental approach. 4 ed. Mountain View: Mayfield; 2005.

11.Padilha KM, Gallani MC, Colombo RC.Development of an instrument to measure beliefs and attitudes from heart valve disease patients. Rev. Latino-Am. Enfermagem. 2004;12(3):453-9.

12.Padilha KM, Gallani MC, Colombo RC. Validity of an instrument to measure the impact of valve heart disease on the patient's daily life. J Clin Nurs. 2007;16(7):1285-91.

13.Cornélio ME, Gallani MC, Godin G, Rodrigues RC, Mendes RD, Nadruz Junior W. Development and reliability of an instrument to measure psychosocial determinants of salt consumption among hypertensive patients. Rev. Latino-Am. Enfermagem. 2009;17(5):701-7.

14.Miura CT, Gallani MC, de Barros Leite Domingues G, Rodrigues RC, Stoller JK. Cultural adaptation and reliability analysis of the Modified Dyspnea Index for the Brazilian culture. Rev. Latino-Am. Enfermagem. 2010;18(5):1020-30.

15. He FJ, MacGregor GA. Importance of salt in determining blood pressure in children. Meta-analysis of Randomized Controlled Trials. Hypertension. 2006;48(11):861-9.

16. Cook NR, Cutler JA, Obarzanek E, Buring JE, Rexrode KM, Kumanyika SK, et al. Long term effects of dietary sodium reduction on cardiovascular disease outcomes: observational follow-up of the Trials of Hypertension Prevention (TOHP). Brit Med J. 2007;334:885-8.

17. He FJ, MacGregor GA. Reducing population salt intake worldwide: from evidence to implementation. Prog Cardiovasc Dis. 2010;52:363-82.

18. Danaei G, Ding EL, Mozaffarian D, Taylor B, Rehm J, Murray CJ, Ezzati M. The preventable causes of death in the United States: comparative risk assessment of dietary, lifestyle, and metabolic risk factors. PLoS Med. 2009;6(4):e10000582009.

19. Ferreira-Sae MC, Gallani MC, Nadruz W, Rodrigues RC, Franchini KG, Cabral PC, Sales ML. Reliability and validity of a semi-quantitative FFQ for sodium intake in low-income and low-literacy Brazilian hypertensive subjects. Public Health Nutr. 2009;12(11):2168-73.

20. Cornélio ME, Gallani MC, Godin G, Rodrigues RC, Nadruz W Jr, Mendez RD. Behavioural determinants of salt consumption among hypertensive individuals. J Hum Nutr Diet. 2012;25(4):334-44.

21. Ajzen, I. The theory of planned behavior: Reactions and reflections. Psychology \& Health. 2011;26(9):1113-27.

22. Cornélio ME; Gallani MC, Agondi RF, Rodrigues RC. Development of the SALdável Program Intervention to Reduce Salt Intake among Hypertensive Brazilian Women: an Intervention Mapping approach. Eur J Cardiovasc Nurs. 2012. In press.

23. Cornélio ME. Impacto de intervenção para fortalecimento da motivação para reduzir o consumo de sal entre mulheres com hipertenção arterial [tese de doutorado]. Campinas (SP): Faculdade de Ciências Médicas da Universidade Estadual de Campinas; 2011.

24. Agondi R de F, Gallani MC, Cornélio ME, Rodrigues RC. Analysis of action plans and coping plans for reducing salt consumption among women with hypertension. Rev. Latino-Am. Enfermagem. 2012 Jun;20(3):486-94. 
25. Gollwitzer PM, Sheeran P, Trötschel R, Webb TL. Selfregulation of priming effects on behavior. Psychol Sci. 2011 Jul;22(7):901-7. 